\title{
Uniform Convergence of Some Extremal Polynomials in Domain with Corners on the Boundary
}

\author{
M. Kucukaslan, C. Koşar, and F. G. Abdullayev \\ Department of Mathematics, Faculty of Science and Literature, Mersin University, 33343 Mersin, Turkey \\ Correspondence should be addressed to F. G. Abdullayev, fabdul@mersin.edu.tr \\ Received 8 December 2009; Accepted 26 March 2010 \\ Academic Editor: Yuming Xing \\ Copyright (c) 2010 M. Kucukaslan et al. This is an open access article distributed under the Creative \\ Commons Attribution License, which permits unrestricted use, distribution, and reproduction in \\ any medium, provided the original work is properly cited. \\ The aim of this paper is to investigate approximation properties of some extremal polynomials \\ in $A_{p}^{1}, p>0$ space. We are interested in finding approximation rate of extremal polynomials to \\ Riemann function in $A_{p}^{1}$ and $C$-norms on domains bounded by piecewise analytic curve.
}

\section{Problem and Main Results}

Let $G$ be a finite region with $z_{0} \in G$ bounded by Jordan curve $L:=\partial G$ and let $w=\varphi(z)$ be the canonical conformal mapping of $G$ onto the disc $D_{r_{0}}:=\left\{w:|w|<r_{0}\right\}$ with $\varphi\left(z_{0}\right)=0, \varphi^{\prime}\left(z_{0}\right)=$ 1 , where $r_{0}$ is called the conformal radius of $\bar{G}$ with respect to $z_{0}$.

Denote by $A_{p}^{1}(G), p \in(0, \infty)$ the set of functions $f(z)$ analytic in $G$ with $f\left(z_{0}\right)=$ $0, f^{\prime}\left(z_{0}\right)=1$ such that

$$
\|f\|_{A_{p}^{1}}=\left\|f^{\prime}\right\|_{A_{p}(G)}:=\left(\iint_{G}\left|f^{\prime}(z)\right|^{p} d \sigma_{z}\right)^{1 / p}<\infty,
$$

where $d \sigma_{z}$ is two-dimensional Lebesgue measure.

Also, let us denote by $\wp_{n}$ the class of all polynomials $P_{n}(z), \operatorname{deg} P_{n} \leq n$, with $P_{n}\left(z_{0}\right)=$ $0, P_{n}^{\prime}\left(z_{0}\right)=1$ and consider following extremal problem:

$$
\iint_{G}\left|\varphi^{\prime}(z)-P_{n}^{\prime}(z)\right|^{p} d \sigma_{z} \longrightarrow \min , \quad p>0
$$


Using a method given in [1, page 137], it is seen that the solution of the extremal problem in (1.2) exists, and if $p>1$, the solution is unique [1, page 142]. This unique solution was denoted by $B_{n, p}(z)$ and it was called $p$-Bieberbach polynomials in [2].

Let us denote the best approximation to $f$ in the class $\wp_{n}$ by $A_{p}^{1}$-norm and $C$-norm by

$$
\begin{gathered}
E_{n}\left(f, A_{p}^{1}\right):=\inf _{P_{n} \in \mathscr{q}_{n}}\left\|f-P_{n}\right\|_{A_{p}^{1}}, \\
E_{n}(f, \bar{G}):=\inf _{P_{n} \in \mathfrak{\xi}_{n}}\left\|f-P_{n}\right\|_{C}=\inf _{P_{n} \in \mathfrak{q}_{n}} \max _{z \in \bar{G}}\left|f(z)-P_{n}(z)\right|,
\end{gathered}
$$

respectively.

It is clear from the definition of $p$-Bieberbach polynomials that

$$
E_{n}\left(\varphi, A_{p}^{1}\right)=\left\|\varphi-B_{n, p}\right\|_{A_{p}^{1}}
$$

One of the problem in approximation theory is to calculate $E_{n}(f, \bar{G})$ through the calculation of $E_{n}\left(f, A_{p}^{1}\right)$ for given $f$. This idea goes back at least as far as in [3, pages 116141].

The special case $p=2$ in (1.2) has two important properties. First, $B_{n, 2}(z)$ coincides with usual Bieberbach polynomials $B_{n}(z)$ and it has an explicit representation via orthogonal polynomials [4]. Second, $B_{n, 2}(z)$ is a main tool in the construction of Riemann mapping function for the given region.

Especially, approximation properties of Bieberbach polynomials were first investigated by Keldych in 1939 in [5], and then considerable progress in this area has been achieved by Mergelyan [6], Suetin [7], Simonenko [8], Andrievski1 [9, 10], Gaier [11, 12], Abdullayev [13-15], Israfilov [16, 17], and the others.

Besides this, approximation properties of $B_{n, p}(z)$ have been investigated only by authors of [2].

In this study, we are going to investigate the problem mentioned above in the region bounded by piecewise analytic curve and consider analytic curve as the image of a segment $[0,1]$ under conformal mapping in a neighborhood of this segment.

Definition 1.1. (a) The curve $L:=\partial G$ is called piecewise analytic if it is a union of finite number of analytic arcs and it has $\lambda_{j} \pi,\left(0<\lambda_{j}<2, j=1,2, \ldots, m\right)$ exterior angles with respect to $G$ on the $z_{j}, j=1,2, \ldots, m$ corners where two arcs meet.

(b) One denotes the class of piecewise analytic curve by $A(\lambda)$ where $\lambda:=\min _{1 \leq j \leq m}\left\{\lambda_{j}\right\}$.

(c) One says $G \in A(\lambda), 0<\lambda<2$, if $L:=\partial G \in A(\lambda), 0<\lambda<2$.

For any $\lambda, 0<\lambda<2$ and $p, 1<p<2 /\left(1-\lambda_{*}\right)$, let one denote

$$
\begin{aligned}
\lambda^{*}:=\max \{1, \lambda\}, \quad \lambda_{*} & :=\min \{1, \lambda\}, \quad \gamma:=\gamma(\lambda ; p)=\frac{\lambda(\lambda-1)}{2-\lambda}+\frac{2}{p} \lambda, \\
\alpha(\lambda) & :=\max \left\{1, \frac{2(1-\lambda)(2-\lambda)}{1+(1-\lambda)^{2}}\right\} .
\end{aligned}
$$


Theorem 1.2. Let $G \in A(\lambda)$ for some $\lambda, 0<\lambda<2$ and $p, 1<p<2 /\left(1-\lambda_{*}\right)$. Then, for any $n=1,2, \ldots$, the $p$-Bieberbach polynomials $B_{n, p}$ satisfy

$$
\left\|\varphi-B_{n, p}\right\|_{A_{p}^{1}} \leq \mathrm{const} \cdot n^{-\gamma}
$$

where $\gamma$ is as in (1.6).

Theorem 1.3 (main theorem). Let $G \in A(\lambda)$ for some $\lambda, 0<\lambda<2$. Then, for any $n=1,2, \ldots$ the $p$-Bieberbach polynomials $B_{n, p}$ satisfy

$$
\left\|\varphi-B_{n, p}\right\|_{C} \leq \mathrm{const} \begin{cases}n^{-\gamma}, & 2<p<\frac{2}{1-\lambda_{*}} \\ n^{-\gamma} \log n, & p=2, \\ n^{-\gamma+(2 / p-1) \lambda^{*},} & \alpha(\lambda)<p<2,\end{cases}
$$

where $\lambda_{*}, \lambda^{*}$, and $\alpha(\lambda)$ are defined in (1.6) and (1.7), respectively.

Corollary 1.4. (a) If the region is a square, then Theorems 1.2 and 1.3 are true for

$$
\gamma=3\left(\frac{1}{2}+\frac{1}{p}\right)
$$

when $1<p<\infty$.

(b) If the region is an L-shaped region then Theorems 1.2 and 1.3 are true for

$$
r=-\frac{1}{6}+\frac{1}{p}
$$

when $6 / 5<p<4$.

Remark 1.5. If we take $p=2$ in Theorems 1.2 and 1.3, we obtain the result of Gaier in [18].

\section{Integral Representation of $\varphi$}

We are going to follow the analog used by Andrievskir and Gaier in [19]. Let us suppose that $\tau_{i}$ is a conformal mapping in an open neigborhood of $[0,1]$ such that $L_{i}:=\tau_{i}([0,1])$. Then, there is a symmetric lens-shaped domain $S_{i}$ whose closure is contained in this open neigborhood of $[0,1]$ (for more information see [19]).

So, we obtain

$$
\tilde{G}:=G \cup\left(\bigcup_{i=1}^{m} \tau_{i}\left(S_{i}\right)\right)
$$


and $\varphi$ can be extended into $\tilde{G}$ as follows:

$$
\tilde{\varphi}(z):= \begin{cases}\varphi(z), & z \in \bar{G}, \\ \overline{\overline{\varphi\left[\tau_{i} \overline{\left(\tau_{i}^{-1}(z)\right)}\right]}}, & z \in \tau_{i}\left(S_{i}\right) \backslash G .\end{cases}
$$

From the construction of $\tilde{G}$, it is clear that $\partial \tilde{G}$ consists of $m$ analytic arc $\Gamma_{i}, i=$ $1,2, \ldots, m$, and $z_{1}, z_{2}, \ldots, z_{m}$ are the common end points of $L_{i}$ and $\Gamma_{i}$.

For an arbitrary small $\varepsilon, \varepsilon<1$, let us choose $R=1+c n^{\varepsilon-1}$ such that $1<R<2$, the points $z_{i}^{(j)}, i=1, \ldots, m, j=1,2$ being the intersection of $\Gamma_{i}$ and $L_{R}$. So, these points divide $\Gamma_{i}$ into three parts such that

$$
\Gamma_{i}=\Gamma_{i}^{1} \cup \Gamma_{i}^{2} \cup \Gamma_{i}^{3}
$$

where

$$
\Gamma_{i}^{1}:=\Gamma_{i}\left(z_{i+1}, z_{i}^{(2)}\right), \quad \Gamma_{i}^{2}:=\Gamma_{i}\left(z_{i}^{(2)}, z_{i}^{(1)}\right), \quad \Gamma_{i}^{3}:=\Gamma_{i}\left(z_{i}^{(1)}, z_{i}\right),
$$

so that

$$
\partial \tilde{G}=\bigcup_{i=1}^{m} \bigcup_{j=1}^{3} \Gamma_{i}^{j}
$$

From the Cauchy integral formula, we have for all $z \in \bar{G}$

$$
\begin{aligned}
\varphi(z) & =\frac{1}{2 \pi i} \int_{\partial \widetilde{G}} \frac{\varphi(t)}{t-z} d t=\frac{1}{2 \pi i} \sum_{i=1}^{m} \sum_{j=1}^{3} \int_{\Gamma_{i}^{j}} \frac{\varphi(t)}{t-z} d t \\
& =\sum_{i=1}^{m}\left(J_{i}^{(1)}+J_{i}^{(2)}+J_{i}^{(3)}\right),
\end{aligned}
$$

where

$$
J_{i}^{(1)}:=\frac{1}{2 \pi i} \int_{\Gamma_{i}^{1}} \frac{\varphi(t)}{t-z} d t, \quad J_{i}^{(3)}:=\frac{1}{2 \pi i} \int_{\Gamma_{i}^{3}} \frac{\varphi(t)}{t-z} d t, \quad J_{i}^{(2)}:=\frac{1}{2 \pi i} \int_{\Gamma_{i}^{2}} \frac{\varphi(t)}{t-z} d t
$$

\section{Some Auxiliary Results}

We will use the notation $a<b$ for $a<c b$, where $c$ is a constant independent from $n$. The following lemma plays central role in proving the main theorem. 
Lemma 3.1. Let $G \in A(\lambda)$ for some $\lambda, 0<\lambda<2$ and let $1<p<2 /\left(1-\lambda_{*}\right)$. Then, for any $n=1,2, \ldots$, there is a polynomial $Q_{n}(z)$ which satisfies both $Q_{n}\left(z_{0}\right)=0$ and

$$
\left\|\varphi-Q_{n}\right\|_{A_{p}^{1}} \prec \frac{1}{n^{r}}
$$

where

$$
\gamma=\lambda\left(\frac{\lambda-1}{2-\lambda}+\frac{2}{p}\right)
$$

Proof. Since " $J_{i}^{(2)}(z), i=1, \ldots, m$ " is analytic on $\bar{G}$, there exists a polynomial with $\operatorname{deg} p_{n-1} \leq$ $n-1[1$, page 142] such that

$$
\left|\left(J_{i}^{(2)}(z)\right)^{\prime}-p_{n-1}(z)\right| \leq \frac{c}{n^{\prime}}, \quad i=1,2, \ldots, m
$$

where $c$ is a constant independent from $n$.

Let us define $Q_{n}(z):=\int_{z_{0}}^{z} p_{n-1}(t) d t$. Then, $Q_{n}\left(z_{0}\right)=0$, and from (2.6) and (3.3) we have

$$
\left|\varphi^{\prime}(z)-Q_{n}^{\prime}(z)\right| \leq \frac{c m}{n}+\sum_{i=1}^{m}\left(\left|\left(J_{i}^{(1)}(z)\right)^{\prime}\right|+\left|\left(J_{i}^{(3)}(z)\right)^{\prime}\right|\right)
$$

By taking integral over $G$ of the $p$ th power of both sides of (3.4), we obtain

$$
\iint_{G}\left|\varphi^{\prime}(z)-Q_{n}^{\prime}(z)\right|^{p} d \sigma_{z} \prec \frac{1}{n^{p}}+\sum_{i=1}^{m}\left(\iint_{G}\left|\left(J_{i}^{1}(z)\right)^{\prime}\right|^{p} d \sigma_{z}+\iint_{G}\left|\left(J_{i}^{3}(z)\right)^{\prime}\right|^{p} d \sigma_{z}\right) .
$$

$J_{i}^{(1)}(z)$ and $J_{i}^{(3)}(z)(i=1,2, \ldots, m)$ have the same property in $\bar{G}$, therefore, it is sufficient to show that $A_{p}^{1}$-norms of $J_{i}^{(1)}(z)$ and $J_{i}^{(3)}(z)$ tend to zero. So, we can restrict our attention only to the estimate of

$$
\iint_{G}\left|\int_{l} \frac{\varphi(t)}{(t-z)^{2}} d t\right|^{p} d \sigma_{z} \longrightarrow 0
$$

where $l=\Gamma_{i}^{(1)}$ or $\Gamma_{i}^{(3)},(i=1,2, \ldots, m)$.

To estimate this term, we need to know the behaviour of $\varphi(t)$ in the neigboorhood of the corner. For this, the main tool is the Lehman result.

We have from [20]

$$
|\varphi(t)| \leq C\left|t-z_{i}\right|^{\alpha_{i}}, \quad t \rightarrow z_{i}, i=1, \ldots, m,
$$

where $\alpha_{i}=1 /\left(2-\lambda_{i}\right), i=1,2, \ldots, m$. 
We conclude from (3.7) and (3.6) that

$$
\begin{aligned}
\iint_{G}\left|\int_{l} \frac{|\varphi(t)|}{|t-z|^{2}} d t\right|^{p} d \sigma_{z} & \prec \iint_{G}\left(\int_{l} \frac{|\varphi(t)|}{|t-z|^{2}} d t\right)^{p} d \sigma_{z} \prec \iint_{G}\left(\int_{l} \frac{\left|t-z_{i}\right|^{\alpha_{i}}}{|t-z|^{2}} d t\right)^{p} d \sigma_{z} \\
& \prec \iint_{G_{1}}\left|\int_{l} \frac{\left|t-z_{i}\right|^{\alpha_{i}}}{|t-z|^{2}} d t\right|^{p} d \sigma_{z}+\iint_{G_{2}}\left(\int_{l} \frac{\left|t-z_{i}\right|^{\alpha_{i}}}{|t-z|^{2}} d t\right)^{p} d \sigma_{z}
\end{aligned}
$$

where

$$
\begin{gathered}
G_{1}:=\left\{z:\left|z-z_{i}\right| \leq \delta_{R}\right\} \cap G, \quad G_{2}:=\left\{z:\left|z-z_{i}\right|>\delta_{R}\right\} \cap G, \\
\delta_{R}:=\left|z_{i}^{(j)}-z_{i}\right|, \quad j=1,2 .
\end{gathered}
$$

If $z \in G_{1}$, we have $|t-z| \sim\left|t-z_{i}\right|+\left|z-z_{i}\right|$. Let us denote $\left|t-z_{i}\right|$ and $\left|z-z_{i}\right|$ with $s, r$, respectively. So,

$$
\begin{aligned}
\iint_{G_{1}}\left(\int_{l} \frac{\left|t-z_{i}\right|^{\alpha_{i}}}{|t-z|^{2}}|d t|\right)^{p} d \sigma_{z} & \leq c_{3} \int_{0}^{\delta_{R}} r\left|\int_{0}^{c_{4} \delta_{R}} \frac{s^{\alpha_{i}}}{(s+r)^{2}} d s\right|^{p} d r \\
& \leq c_{3} \int_{0}^{\delta_{R}} r\left|\int_{0}^{r} \frac{s^{\alpha_{i}}}{r^{2}} d s+\int_{r}^{c_{4} \delta_{R}} s^{\alpha_{i}-2} d s\right|^{p} d r \\
& \leq c_{3} \int_{0}^{\delta_{R}} r\left(\frac{r^{\alpha_{i}+1}}{r^{2}}+c_{5} \delta_{R}^{\alpha_{i}-1}-r^{\alpha_{i}-1}\right)^{p} d r \\
& \leq \int_{0}^{\delta_{R}} r \delta_{R}^{p\left(\alpha_{i}-1\right)} d r \leq c_{6} \delta_{R}^{p\left(\alpha_{i}-1\right)+2}
\end{aligned}
$$

for $p\left(\alpha_{i}-1\right)+2>0$.

If $z \in G_{2}$, we have $|t-z| \sim\left|z-z_{i}\right|$. So,

$$
\begin{aligned}
\iint_{G_{2}}\left|\int_{l} \frac{\left|t-z_{i}\right|^{\alpha_{i}}}{|t-z|^{2}}\right| d t||^{p} d \sigma_{z} & \leq \iint_{G_{2}}\left|\int_{l} \frac{\left|t-z_{i}\right|^{\alpha_{i}}}{\left|z-z_{i}\right|^{2}}\right| d t||^{p} d \sigma_{z} \leq \iint_{G_{2}} \frac{\delta_{R}^{\left(\alpha_{i}+1\right) p}}{|z|^{2 p}} d \sigma_{z} \\
& \leq c \delta_{R}^{\left(\alpha_{i}+1\right) p} \int_{\delta_{R}}^{\infty} r^{1-2 p} d r \leq \delta_{R}^{p\left(\alpha_{i}-1\right)+2} .
\end{aligned}
$$

Substituting (3.10) and (3.11) into (3.6), we obtain

$$
\iint_{G}\left|\int_{l} \frac{\varphi(t)}{(t-z)^{2}} d t\right|^{p} d \sigma_{z} \leq \delta_{R}^{p\left(\alpha_{i}-1\right)+2},
$$

and also from (3.5), we have

$$
\left\|\varphi-Q_{n}\right\|_{A_{p}^{1}}^{p} \leq \delta_{R}^{p\left(\alpha_{i}-1\right)+2}
$$


Journal of Inequalities and Applications

If we use Lehman result [20] for $\Psi=\Phi^{-1}$, we obtain

$$
\delta_{R}:=\left|z_{i}^{(j)}-z_{i}\right|=\left|\Psi\left(\Phi\left(z_{i}^{(j)}\right)\right)-\Psi\left(\Phi\left(z_{i}\right)\right)\right| \leq\left|\Phi\left(z_{i}^{(j)}\right)-\Phi\left(z_{i}\right)\right|^{\lambda_{i}} \leq n^{(\epsilon-1) \lambda_{i}} .
$$

The proof is completed by (3.13) and (3.14).

Lemma 3.2. Let $G \in A(\lambda), 0<\lambda<2$. Then, for all polynomials $P_{n}(z), \operatorname{deg} P_{n}(z) \leq n$ with $P_{n}\left(z_{0}\right)=0, n=2,3, \ldots$, one has

$$
\left\|P_{n}\right\|_{C} \prec\left\|P_{n}\right\|_{A_{p}^{1}} \begin{cases}1, & p>2 \\ \sqrt{\log n}, & p=2 \\ n^{(2 / p-1) \lambda^{*}}, & p<2 .\end{cases}
$$

Proof. We will prove only the case $p<2$ since the other cases are already given in $[10,21]$.

Let $z$ be an arbitrary fixed point on the boundary. It is clear from [14, Lemma 2.2] that $l\left(z_{0}, z\right) \subset G$ exists joining $z_{0}, z$ and satisfying cord arc properties. If $l_{1}:=\left\{\xi \in l\left(z_{0}, z\right):|\xi-z| \leq\right.$ $\left.\varepsilon n^{-\lambda_{*}}\right\}$ and $l_{2}:=l\left(z_{0}, z\right) \backslash l_{1}$, then we have

$$
\left|P_{n}(z)\right|=\left|\int_{l\left(z_{0}, z\right)} P_{n}^{\prime}(\xi) d \xi\right| \leq \int_{l_{1}}\left|P_{n}^{\prime}(\xi)\right||d \xi|+\int_{l_{2}}\left|P_{n}^{\prime}(\xi)\right||d \xi|
$$

It is well known from [14, Corollary 2.3] that

$$
\left\|P_{n}^{\prime}\right\|_{C(\bar{G})} \leq c_{1} n^{\lambda^{*}} \cdot\left\|P_{n}\right\|_{C(\bar{G})} .
$$

At the same time, mes $\left(l_{1}\right) \leq c_{2} \varepsilon n^{-\lambda^{*}}$ is valid for a positive constant $c_{2}$ which is independent from $\varepsilon$. Using the Mean Value property of subharmonic function $\left|P_{n}^{\prime}(\xi)\right|^{p}$ (see [22, page 482]), we have for arbitrary point $\xi \in l_{2}$

$$
\left|P_{n}^{\prime}(\xi)\right| \leq \frac{1}{\left[\pi d^{2}(\xi, L)\right]^{1 / p}}\left\|P_{n}\right\|_{A_{p}^{1},}
$$

and after combining (3.18) and (3.16), we obtain

$$
\begin{aligned}
\left|P_{n}(z)\right| & \leq c_{1} n^{\lambda^{*}} \cdot\left\|P_{n}\right\|_{C(\bar{G})} \int_{l_{1}}|d \xi|+c_{3}\left\|P_{n}\right\|_{A_{p}^{1}} \int_{l_{2}} \frac{|d \xi|}{d^{2 / p}(\xi, L)} \\
& \leq c_{1} n^{\lambda^{*}} \cdot\left\|P_{n}\right\|_{C(\bar{G})} \cdot c_{2} \varepsilon n^{-\lambda^{*}}+c_{3}\left\|P_{n}\right\|_{A_{p}^{1}} \int_{l_{2}} \frac{|d \xi|}{|\xi-z|^{2 / p}} \\
& \leq c_{1} c_{2} \varepsilon\left\|P_{n}\right\|_{C(\bar{G})}+c_{3}\left\|P_{n}\right\|_{A_{p}^{1}} \int_{c_{2} \varepsilon n^{-\lambda^{*}}}^{\operatorname{mes}(l)} \frac{d t}{t^{2 / p}} \\
& \leq c_{1} c_{2} \varepsilon\left\|P_{n}\right\|_{C(\bar{G})}+c_{3}\left\|P_{n}\right\|_{A_{p}^{1} n^{(2 / p-1) \lambda^{*}}}
\end{aligned}
$$



is obtained.

Using the maximum modulus principle and choosing $\varepsilon$ satisfying $c_{1} c_{2} \varepsilon<1$, the proof

Lemma 3.2 shows how we can measure $C$-norm of polynomials by using its $A_{p}^{1}$-norm.

Lemma 3.3 (see [2]). Let $G \subset \mathbb{C}$ be a simply connected region so that

$$
\left\|\varphi-B_{n, p}\right\|_{A_{p}^{1}} \leq n^{-\eta}
$$

for each $\mu \in(0,1), n=1,2, \ldots$, and

$$
\left\|P_{n}\right\|_{C} \prec n^{\mu}\left\|P_{n}\right\|_{A_{p}^{1}}
$$

for all polynomials $P_{n}(z), \operatorname{deg} P_{n} \leq n$ with $P_{n}\left(z_{0}\right)=0$. Then,

$$
\left\|\varphi-B_{n, p}\right\|_{C} \leq n^{\mu-\eta}
$$

\section{Proof of Theorems 1.2 and 1.3}

\subsection{Proof of Theorem 1.2}

Let us set $P_{n}(z)$ as follows:

$$
P_{n}(z):=Q_{n}(z)+\left(\varphi^{\prime}\left(z_{0}\right)-Q_{n}^{\prime}(z)\right)\left(z-z_{0}\right)
$$

where $Q_{n}(z)$ as in Lemma 3.1 and satisfying $Q_{n}\left(z_{0}\right)=0$.

It is clear from the definition of $P_{n}(z)$ that $P_{n}\left(z_{0}\right)=0, P_{n}^{\prime}\left(z_{0}\right)=1$ is satisfying

$$
\left|\varphi^{\prime}(z)-P_{n}^{\prime}(z)\right| \leq\left|\varphi^{\prime}(z)-Q_{n}^{\prime}(z)\right|+\left|\varphi^{\prime}\left(z_{0}\right)-Q_{n}^{\prime}\left(z_{0}\right)\right| .
$$

So, we have

$$
\left\|\varphi-P_{n}\right\|_{A_{p}^{1}}^{p} \leq \delta_{R}^{p\left(\alpha_{i}-1\right)+2}+\left|\varphi^{\prime}\left(z_{0}\right)-Q_{n}^{\prime}\left(z_{0}\right)\right|
$$

and from the Mean Value Theorem in [4] we also have

$$
\left|\varphi^{\prime}\left(z_{0}\right)-Q_{n}^{\prime}\left(z_{0}\right)\right| \leq \frac{1}{\pi d^{2 / p}\left(z_{0}, L\right)}\left\|\varphi-Q_{n}\right\|_{A_{p}^{1}}
$$

So, (4.3), (4.4), and (3.13) give

$$
\left\|\varphi-P_{n}\right\|_{A_{p}^{1}}^{p} \leq n^{\left.-\left(p\left(\alpha_{i}-1\right)+2\right)\right)} .
$$

Using extremal properties of the $p$-Bieberbach polynomials, the proof is completed. 


\subsection{Proof of Theorem 1.3}

Lemma 3.3 shows that it is enough to choose $\eta, \mu$ in (3.20) and (3.21), respectively. For this, we take $\eta$ as in Theorem 1.2 and $\mu$ as in Lemma 3.2.

\section{References}

[1] P. J. Davis, Interpolation and Approximation, Blaisdell, Waltham, Mass, USA, 1963.

[2] M. Kucukaslan and F. G. Abdullayev, "New extremal polynomials and its approximation properties," Novi Sad Journal of Mathematics, vol. 39, no. 2, pp. 21-34, 2009.

[3] J. L. Walsh, Interpolation and Approximation by Rational Functions in the Complex Domain, American Mathematical Society, Providence, RI, USA, 1960.

[4] D. Gaier, Lectures on Complex Approximation, Birkhäuser, Boston, Mass, USA, 1987.

[5] M. V. Keldych, "Sur l'approximation en moyenne quadratique des fonctions analtiques," Mathematics Sbornik, vol. 5, pp. 391-400, 1939.

[6] S. N. Mergelyan, "Certain questions of the constructive theory of functions," Proceedings of the Steklov Institute of Mathematics, vol. 37, pp. 3-91, 1951.

[7] P. K. Suetin, Polynomials Orthogonal over the Region and Bieberbach Polynomial, American Mathematical Society, Providence, RI, USA, 1974.

[8] I. B. Simonenko, "On the convergence of bieberbach polynomials in the case of lischitz domain," Mathematics of the USSR-Izvestiya, vol. 13, pp. 166-174, 1979.

[9] V. V. Andrievskiı̌, "On the convergence of Bieberbach polynomials in domains with piecewisequasiconformal boundary," in Theory of Mappings and Approximation of Functions, pp. 3-18, Naukova Dumka, Kyiv, Ukrania, 1983.

[10] V. V. Andrievski1,, "Convergence of Bieberbach polynomials in domains with a quasiconformal boundary," Ukrainian Mathematical Journal, vol. 35, no. 3, pp. 223-236, 1983.

[11] D. Gaier, "On the convergence of the Bieberbach polynomials in regions with corners," Constructive Approximation, vol. 4, no. 3, pp. 289-305, 1988.

[12] D. Gaier, "Polynomial approximation of conformal maps," Constructive Approximation, vol. 14, no. 1, pp. 27-40, 1998.

[13] F. G. Abdullayev, "On the convergence of Bieberbach polynomials in domains with interior zero angels," Doklady Akademii Nauk Ukraine, SSR. Series. A, vol. 12, pp. 3-5, 1989.

[14] F. G. Abdullayev, "Uniform convergence of the generalized Bieberbach polynomials in regions with non-zero angles," Acta Mathematica Hungarica, vol. 77, no. 3, pp. 223-246, 1997.

[15] F. G. Abdullayev, "Uniform convergence of the Bieberbach polynomials inside and on the closure of domains in the complex plane," East Journal on Approximations, vol. 7, no. 1, pp. 77-101, 2001.

[16] D. M. Israfilov, "Approximation by $p$-Faber polynomials in the weighted Smirnov class $E^{p}(G, w)$ and the Bieberbach polynomials," Constructive Approximation, vol. 17, no. 3, pp. 335-351, 2001.

[17] D. M. Israfilov, "Uniform convergence of the Bieberbach polynomials in closed smooth domains of bounded boundary rotation," Journal of Approximation Theory, vol. 125, no. 1, pp. 116-130, 2003.

[18] D. Gaier, "On the convergence of the Bieberbach polynomials in regions with piecewise analytic boundary," Archiv der Mathematik, vol. 58, no. 5, pp. 462-470, 1992.

[19] V. V. Andrievskiı̌ and D. Gaier, "Uniform convergence of Bieberbach polynomials in domains with piecewise quasianalytic boundary," Mitteilungen aus dem Mathematischen Seminar Giessen, no. 211, pp. 49-60, 1992.

[20] R. S. Lehman, "Development of the mapping function at an analytic corner," Pacific Journal of Mathematics, vol. 7, pp. 1437-1449, 1957.

[21] D. Israfilov, “On the approximation properties of extremal polynomials (Russian)," Department of the VINITI , No. 5461, 1981.

[22] I. I. Privalov, Introduction to the Theory of Functions of a Complex Variable, Naukova Dumka, Kyiv, Ukrania, 1984. 